\title{
L'externalisation des frontières des « Nords » dans les eaux des « Suds »
}

L'exemple des dispositifs frontaliers américains et européens visant au contrôle de l'émigration caribéenne et subsaharienne

"Northern" borders in "Southern" waters: the externalisation of borders and the example of American and European border apparatuses for the control of

Caribbean and Sub-Saharan emigration

\section{Cedric Audebert et Nelly Robin}

\section{(2) OpenEdition \\ 1 Journals}

\section{Édition électronique}

URL : http://journals.openedition.org/conflits/17512

DOI : $10.4000 /$ conflits. 17512

ISSN : $1777-5345$

Éditeur :

CCLS - Centre d'études sur les conflits lilberté et sécurité, L'Harmattan

\section{Édition imprimée}

Date de publication : 30 mars 2009

Pagination : 35-51

ISBN : 978-2-296-07748-5

ISSN : 1157-996X

Référence électronique

Cedric Audebert et Nelly Robin, «L'externalisation des frontières des « Nords » dans les eaux des « Suds » », Cultures \& Conflits [En ligne], 73 I printemps 2009, mis en ligne le 30 mars 2010, consulté le 30 mars 2021. URL : http://journals.openedition.org/conflits/17512 ; DOI : https://doi.org/10.4000/ conflits. 17512 


\title{
L'externalisation des frontières des "Nords" dans les eaux des "Suds". L'exemple des dispositifs frontaliers américains et européens visant au contrôle de l'émigration caribéenne et subsaharienne
}

\section{Cedric AUDEBERT}

Cédric Audebert est chercheur CNRS au laboratoire Migrinter de l'université de Poitiers. Il travaille sur la métropolisation des migrations antillaises aux Etats-Unis et a notamment publié L'Insertion socio-spatiale des Haïtiens à Miami (L'Harmattan, 2003).

\section{Nelly ROBIN}

Nelly Robin est chercheuse du CEPED, bébergée au laboratoire Migrinter de Poitiers. Elle travaille sur la dimension politique des migrations ouest-africaines et a notamment co-dirigé l'Atlas de l'intégration régionale en Afrique de l'Ouest (CEDEAO-CSAO/OCDE, 2006).

\begin{abstract}
u début de l'année 2006, l'opinion internationale s'est émue du naufrage, aux frontières de l'Europe, de milliers d'émigrants clandestins subsahariens, dont le destin tragique rappelle celui des boat people antillais ayant tenté de rejoindre les Etats-Unis au cours des trois dernières décennies. Quoique la dimension politique ait joué un rôle majeur dans la genèse des premiers flux massifs de boat people haïtiens et cubains dans les années 1960, les migrations maritimes subsahariennes et caribéennes répondent à des déterminants similaires. Elles expriment l'acuité de la crise économique sévissant dans les pays d'origine et correspondent à une demande sociale, dans un contexte où la migration de l'individu est perçue comme un préalable nécessaire à l'ascension sociale du collectif familial.
\end{abstract}

Dans les deux cas, la maritimisation croissante de l'émigration répond au durcissement des politiques migratoires des « Nords » ainsi qu’à la mise en place 
de dispositifs frontaliers de plus en plus sophistiqués : en cayucos, les Sénégalais quittent les côtes du Sahara pour rejoindre les terres insulaires du « vieux continent » avec pour devise «Barça... walla Basakb ${ }^{1}$ »; en kanntè, les Haïtiens fuient leur pays avec l'espoir d'atteindre les rivages du «pays où il suffit de se baisser pour ramasser les dollars ». Mais une même réponse leur est opposée par l'Europe et les Etats-Unis : des mesures répressives qui conduisent à traiter la migration comme un acte criminel et le migrant comme un délinquant.

Aujourd'hui, les pays européens tendent à promouvoir l'idée d'une approche nouvelle des migrations, associant des initiatives de prévention et des projets de réinsertion, étroitement liés aux politiques d'aide au développement. Toutefois, les dispositifs maritimes mis en place le long des côtes sénégalaises expriment surtout la volonté des pays européens d'externaliser le contrôle de leurs frontières, reproduisant en cela une stratégie états-unienne bien connue dans la Caraïbe. A vingt-cinq ans d'écart et à des milliers de kilomètres de distance, cette similitude de choix politique s'incarne dans ce que l'on considère aujourd'hui comme une externalisation des frontières des pays du Nord.

En réalité, le terme «externalisation ", comme le verbe « externaliser », sont de plus en plus utilisés par la communauté scientifique. Cette évolution sémantique vise à qualifier la volonté des pays européens comme celle des Etats-Unis de conférer aux pays du Sud une responsabilité croissante de la gestion et du contrôle des frontières des pays du Nord. Cette terminologie, empruntée à l'économie, et plus spécifiquement à l'environnement de l'entreprise, est ainsi transposée dans d'autres disciplines telles que la géographie ou l'analyse politique. Le sens qui lui est alors donné est admis implicitement sans qu'une définition précise, adaptée au nouveau contexte dans lequel il est employé, ne soit proposée. Une mise en perspective des dynamiques migratoires caribéennes et subsahariennes peut aider à comprendre cette transposition de vocabulaire de l'économie à la géopolitique. Elle peut aussi aider à mieux cerner les implications des politiques en question sur la dynamique des flux et notamment la diversification des stratégies spatiales.

\section{L'externalisation des frontières des « Nords » : la transposition géopolitique d'une notion économique}

Les économistes définissent l'externalisation comme «le recours à un prestataire ou à un fournisseur externe, pour une activité qui était jusqu'alors réalisée au sein de l'entreprise ", celle-ci étant généralement assortie d'un «transfert de ressources matérielles ou humaines vers le prestataire choisi 2 ». Dans le cadre d'une réflexion sur la pertinence de l'application de cette notion

1. «Barcelone ou la mort».

2. Quélin B., Duhamel A., «Externalisation : logique financière et logique de compétence sont liées ", Problèmes économiques, n²799, 2003, pp. 8-10. 
aux relations internationales et à la géographie, il convient au préalable de s'interroger sur les raisons et les logiques de ce processus - en lien avec l'évolution du contexte international - ainsi que sur ses formes.

De même que la concurrence liée à l'ouverture des marchés nécessite de la part des acteurs économiques la mise en place de stratégies transnationales, la mondialisation impulse l'accélération, la massification et la diversification des mobilités humaines en même temps qu'elle redéfinit et complexifie des identités qui concurrencent parfois les référents stato-nationaux. Dans ce contexte de mobilités multiformes croissantes, l'immigration est de plus en plus perçue par les sociétés nationales du Nord comme un phénomène qui leur échappe, en particulier lorsqu'elle concerne des populations migrantes dont l'altérité construite est représentée - à tort ou à raison - comme un risque pour la cohésion de ces sociétés.

Les migrations subsahariennes et maghrébines vers l'Europe, les migrations caribéennes et latino-américaines vers l'Amérique du Nord et les migrations asiatiques et mélanésiennes vers l'Australie correspondent clairement à ce schéma. En référence à l'immigration maritime caribéenne noire, Reagan déclarait ainsi en 1981 que les demandeurs d'asile étaient devenus un problème national majeur au détriment des intérêts des Etats-Unis, tandis qu'un élu local s'exclamait: "Nous ne voulons plus de satanés réfugiés noirs en Floride! ${ }^{3 » .}$ En réponse aux fantasmes économiques et civilisationnels doutant de la nécessité de l'immigration pour l'économie et pour le dynamisme social et démographique des sociétés d'accueil, les gouvernements s'engagent dans des politiques de contrôle des flux de plus en plus drastiques. Les politiques de coopération « Nord-Sud» sont de plus en plus soumises au double objectif de « sécurisation » des frontières et de fixation des migrants dans leur pays d'origine.

En appui à l'imperméabilisation des frontières, la stratégie d'externalisation de la politique migratoire rentre alors en scène pour transférer à des pays tiers - généralement les pays de transit et d'origine - la gestion de l'immigration « indésirable » pour mieux la contenir, à l'instar des entreprises externalisant une partie de leur activité. Mais l'enjeu est également de sélectionner à distance l'immigration " utile », notamment par la mise en place de camps. La science économique a montré que trois des principaux facteurs motivant l'externalisation des entreprises résidaient dans la baisse des coûts opérationnels, la flexibilité et la recherche de l'amélioration de la qualité de service ${ }^{4}$.

Les économistes mettent également l'accent sur les logiques évolutives de l'externalisation et leur accélération, sur la base des expériences précédentes et 
l'attente forte des entreprises vis-à-vis du professionnalisme des prestataires. Ce processus repose sur deux leviers :

- la délocalisation du capital, des infrastructures, de l'expertise et du savoir-faire ;

- le transfert de responsabilités : obligation de résultats, lien entre transfert de compétences et logiques financières.

La transposition de la notion économique d'externalisation dans le champ géopolitique vise à traduire les implicites des politiques migratoires américaines et européennes ainsi que leur évolution récente. Toutefois, comme le soulignent Quélin et Duhamel, "on ne décrète pas l'externalisation, on la construit ». Il s'agit donc pour nous de comprendre comment ce processus dit d'« externalisation » a été élaboré et mis en place par les Etats-Unis et l'Union européenne aux portes ou sur les territoires des pays caribéens et subsahariens. Il s'agit aussi de s'interroger sur la pertinence de la notion d'" externalisation » à propos de la mise en place des dispositifs frontaliers américains et européens visant au contrôle de l'émigration caribéenne et subsaharienne.

\section{La politique des visas : première étape de l'externalisation de la ges- tion et du contrôle des frontières}

Jusqu'au milieu de la décennie 1980, les émigrés ouest-africains rejoignent les pays du Nord essentiellement par voie aérienne. Toutefois, les années 1990 constituent un tournant: les règles du jeu du système migratoire entre l'Afrique de l'Ouest et l'Europe commencent à changer et ces mutations se poursuivent et s'affirment tout au long de la décennie 19905.

La convention d'application des accords de Schengen entre en vigueur en 1995 , pour les premiers pays signataires ${ }^{6}$; elle « instaure un espace de libre circulation des personnes entre les Etats signataires et associés tout en garantissant une protection renforcée aux frontières extérieures de l'espace ». Dès lors, pour franchir les frontières de l'espace Schengen, les ressortissants des pays tiers à l'Union européenne, auxquels appartiennent les pays ouest-africains, sont soumis à un visa dit « visa Schengen »; il s'agit d'un visa unique délivré par un des Etats membres, valable pour l'ensemble de la zone Schengen ${ }^{7}$.

5 . Bossard L., Gnisci D., Robin N., Trémollières M., «Les Migrations », Atlas de l'intégration régionale en Afrique de l'Ouest, CEDEAO-CSAO/OCDE, août 2006.

6. En 1985, les trois pays du Bénélux, l'Allemagne et la France; en 1990, l'Italie; en 1991, l’Espagne et le Portugal ; en 1992, la Grèce; en 1995, l’Autriche ; puis en 1996, le Danemark, la Finlande et la Suède.

7. Le Royaume-Uni et l'Irlande n'ont pas signé cette Convention mais participent partiellement aux mesures adoptées dans le cadre des accords Schengen. La Norvège, l'Islande et la Suisse, bien 
A peine un an plus tard, ce dispositif est renforcé par la création du visa de transit aéroportuaire (VTA) ${ }^{8}$.

"Considérant que la voie aérienne, [...] lors d'un transit aéroportuaire, constitue un moyen important de pénétration en vue notamment d'un établissement illégal sur le territoire des Etats membres et qu'il convient de rechercher une amélioration de la maitrise de cette voie 9 ",

l'autorisation est donnée aux Etats membres de l'Union de déroger au principe de «libre passage en transit par la zone internationale des aéroports », établi par l'annexe 9 de la convention de Chicago ${ }^{10}$. Seuls les ressortissants des pays tiers sont soumis à ce principe d'exception. En 1996, une première liste de pays est publiée ${ }^{11}$ avec, parmi eux, deux pays ouest-africains : le Ghana et le Nigeria. Entre 2002 et 2008, conformément à l'article 5 de cette action commune qui prévoit que "chaque Etat membre décide s'il y a lieu d'exiger un visa de transit aéroportuaire des ressortissants des pays non mentionnés sur la liste », la France y ajoute le Burkina Faso, la Côte d'Ivoire, la Gambie, la Guinée, la Guinée Bissau, le Liberia, le Mali, le Sénégal et la Sierra Leone 12 notamment. En 2008, ces mesures deviennent encore plus draconiennes pour les ressortissants de la Guinée, du Liberia et de la Sierra Leone dont «les titulaires d'un passeport diplomatique, de service ou officiel 13 ", ou "les titulaires d'un visa valable pour un Etat membre de l'Union européenne ou de l'Espace économique européen, le Canada, les Etats-Unis d'Amérique et la Suisse 14 » ne sont plus dispensés de l'obligation de VTA, contrairement aux ressortissants des autres Etats.

Dès lors, au départ des aéroports internationaux de l'ensemble de ces pays, les exploitants, c'est-à-dire les transporteurs aériens, sont tenus «de

qu'extérieurs à l'Union, sont associées à l'espace Schengen par un accord de coopération avec les pays signataires de la Convention.

8. Le visa de transit aéroportuaire est délivré par les services consulaires des Etats membres.

9. 96/197/JAI : Action commune, du 4 mars 1996, adoptée par le Conseil sur la base de l'article K.3 du traité de l'Union européenne, relative au régime du transit aéroportuaire. Journal officiel $\mathrm{n}^{\circ} \mathrm{L} 063,13$ mars 1996, pp. 008-009.

10. Robin N., «L'émigration internationale à Dakar : au cœur des nouveaux trafics mondiaux », in Lombard J., Mesclier E., Velut S. (dirs), La Mondialisation côté sud. Acteurs et territoires, Paris, ENS/IRD, 2006, pp. 143-162.

11. Afghanistan, Ethiopie, Erythrée, Ghana, Irak, Iran, Nigeria, Somalie, Sri Lanka, Zaïre. 96/197/JAI : Action commune, du 4 mars 1996, adoptée par le Conseil sur la base de l'article K.3 du traité de l'Union européenne, relative au régime du transit aéroportuaire.

12 . JORF n ${ }^{\circ} 0020$ du 24 janvier 2008, texte 24. Arrêté du 15 janvier 2008 fixant la liste des Etats dont les ressortissants sont soumis au visa consulaire de transit aéroportuaire et les exceptions à cette

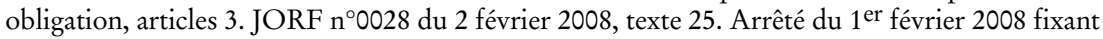
la liste des Etats dont les ressortissants sont soumis au visa consulaire de transit aéroportuaire et les exceptions à cette obligation, article 1 .

13. Arrêté du 15 janvier 2008 fixant la liste des Etats dont les ressortissants sont soumis au visa consulaire de transit aéroportuaire et les exceptions à cette obligation, article 4, alinéa 1 .

14. Ibid., article 4, alinéa 3. 
prendre des précautions au point d'embarquement pour faire en sorte que les passagers soient en possession des documents prescrits par les Etats de transit et de destination aux fins de contrôle 15 ». Ainsi, en s'accordant le droit de déroger aux principes fondamentaux de l'Organisation de l'aviation civile internationale (OACI) pour la première fois, les pays européens se dotent d'outils juridiques pour ériger, aux portes des pays du Sud, les frontières de l'immigration dans les pays du Nord.

Si l'on considère, comme les économistes, qu'il y a externalisation "lorsqu'une entreprise délègue à un prestataire externe à l'entreprise la gestion et l'exécution d'une activité récurrente qu'elle réalisait en interne auparavant ", alors l'instauration du VTA révèle, dès les années 1990, la volonté des pays européens d'externaliser la gestion et le contrôle de leurs frontières à celles des pays du Sud.

Ce dispositif est renforcé par la notion de pays d'origine sûrs, introduite en droit français dès 2003 16. Un pays est considéré comme sûr « s'il veille an respect des principes de liberté, de la démocratie et de l'état de droit, ainsi que des droits de l'Homme et des libertés fondamentales 17 ». Les demandeurs d'asile, ressortissants des Etats concernés, ne peuvent ni bénéficier d'une admission au séjour au titre de l'asile ni percevoir l'allocation temporaire d'attente. La liste actuellement en vigueur comprend quinze Etats ${ }^{18}$ dont cinq Etats ouest-africains : le Bénin, le Cap-Vert, le Ghana, le Mali et le Sénégal.

Les politiques européennes actuelles des visas et d'octroi de l'asile vis-àvis de ces pays rappellent beaucoup celles initiées il y a plus de vingt ans par Washington vis-à-vis des Haïtiens, comme nous le verrons plus loin. En outre, d'autres pays du bassin caribéen dont l'effectif des émigrants aux Etats-Unis reste limité se sont vus, malgré tout, imposer un durcissement des conditions d'entrée. Ils étaient en effet utilisés comme lieux de transit pour d'autres filières migratoires impliquant des volumes bien plus importants. Ainsi, l'acquisition relativement aisée de la citoyenneté dominiquaise sans condition de résidence pour les investisseurs a été utilisée par une filière chinoise pour entrer aux Etats-Unis. En réaction, l'imposition de nouvelles conditions d'entrée pour tous les ressortissants dominiquais a eu pour effet de diviser par trois le volume de leurs entrées dans ce pays entre 2002 et 2003.

Les contrôles rendant difficile l'émigration de la Caraïbe vers le Nord s'inscrivent dans le prolongement de dispositifs états-uniens de lutte contre

15. Paragraphe 3.53, chapitre 3, Annexe 9 de la Convention de Chicago.

16. Loi du 10 décembre 2003, OFPRA.

17. Au sens de l'article L.741-4,2 ${ }^{\circ}$ du Code de l'entrée et du séjour des étrangers et du droit d'asile.

18. Bénin, Bosnie-Herzégovine, Cap-Vert, Croatie, Géorgie, Ghana, Inde, Madagascar, Mali, Macédoine, Maurice, Mongolie, Sénégal, Tanzanie, Ukraine. 
l'immigration ayant largement eu recours à l'externalisation depuis près de trois décennies. A ce stade de l'analyse, il convient de comprendre en quoi la diversification des routes migratoires - assortie de leur clandestinité croissante - constitue une conséquence de la mise en place de ce dispositif en même temps qu'elle a contribué à l'entretenir et à le justifier aux yeux de Washington et des capitales européennes. Nous verrons que dans l'évolution de leur configuration spatiale et dans les réponses politiques qui leurs sont opposées de la part des pays du Nord, les migrations subsahariennes actuelles vers l'Europe présentent un certain nombre de similitudes avec l'expérience des migrations dans la «méditerranée américaine ».

\section{La diversification des routes migratoires et la mise en place d'ac- cords bilatéraux}

Les pays ouest-africains et caribéens traversent des périodes de crise économique, sociale et/ou politique qui favorisent l'augmentation et la diversification du profil des candidats à l'émigration internationale. Une part substantielle d'entre eux cherche des alternatives à la problématique "émigration » par voie aérienne et se tourne vers les voies terrestres et maritimes, faites d'itinéraires complexes et souvent animées par des réseaux de trafic illicite de migrants les enfermant durablement dans la clandestinité. En réponse, les pays du Nord mettent en œuvre un dispositif fondé sur trois piliers : l'interception, l'enfermement et le rapatriement (ou la réadmission).

\section{L'expérience des boat people haïtiens}

$\mathrm{Du}$ fait de ses caractéristiques archipélagiques, la Caraïbe a très tôt été le théâtre de migrations clandestines maritimes de grande ampleur entre les îles et vers les Etats-Unis. Les pêcheurs du Nord d'Haïti s'installent aux Bahamas dès les années 195019 et les premiers boat people dominicains vers Puerto Rico sont enregistrés dès le début des années 1970 20. Avec la répression croissante de l'immigration clandestine après l'indépendance des Bahamas en 1973 et l'appauvrissement de zones rurales en Haïti, les couches populaires ont émigré par bateau directement vers les Etats-Unis ${ }^{21}$. Plus récemment, avec la désagrégation du bloc communiste et l'approfondissement de la crise économique à Cuba, le flux des balseros s'est considérablement développé vers les Etats-Unis et doit faire face à un accueil bien moins favorable que pendant la Guerre froide.

19. Marshall D., “The Haitian problem”, Illegal migration to the Babamas, Cave Hill, Institute of Social and Economic Research, University of the West Indies, 1979.

20. Duany, Jorge, “Dominican migration to Puerto Rico: a transnational perspective”, Centro Journal, vol.17, $\mathrm{n}^{\circ} 1,2005$, p. 247.

21. Audebert C., "Stratégies géopolitiques, dynamiques géoéconomiques et migrations caribéennes : des aires d'influence aux réseaux », in Lambourdière E. (dir.), Les Caraibes dans la géopolitique mondiale, Paris, Ellipses, 2007, pp. 261-281. 
Depuis le début des années 1980, les gardes-côtes états-uniens ont ainsi intercepté plus de 200000 boat people antillais, dont 111000 Haïtiens, 64000 Cubains et 34000 Dominicains ${ }^{22}$. Plusieurs dizaines (centaines ?) de milliers d'autres ont disparu en mer et, derrière ces chiffres, se cachent de véritables tragédies humaines. Au total, plus de $92 \%$ des boat people interceptés par les gardes-côtes sont originaires de ces trois pays de la Caraïbe, vis-à-vis desquels les Etats-Unis ont imaginé un dispositif d'interception et de traitement de la migration qu'ils ont voulu dissuasif, mais sans grand succès.

De ce point de vue, l'expérience des Haïtiens est riche d'enseignements, tant la politique de répression de leur migration est singulière dans l'histoire des Etats-Unis. Tout d'abord, le dispositif d'interception repose sur une vingtaine de vaisseaux patrouillant à proximité des côtes de Floride et de Puerto Rico et ayant une autonomie de 60 jours; et deux vaisseaux-héliports ayant une autonomie de 90 jours leur permettant d'intervenir à proximité des côtes haïtiennes, et dotés de systèmes de détection en haute mer.

Ces moyens viennent en appui de choix politiques ayant pour objectif, depuis trente ans, de contenir l'immigration haïtienne. En 1978, le Haitian Program est mis en place pour déporter massivement les immigrés clandestins ou en instance de régularisation. On passe alors à Miami en quelques semaines de moins de 10 audiences à 150 audiences quotidiennes en vue d'une reconduite rapide en Haïti ${ }^{23}$. Il s'agit de les empêcher d'obtenir l'asile et de dissuader les départs d'Haïti. En 1981, Reagan va plus loin en signant avec le gouvernement haïtien l'Interdiction agreement afin de permettre aux gardes-côtes d'intercepter directement en mer les boat people, d'interroger sur place les demandeurs d'asile et, le cas échéant, de les renvoyer en Haïti.

L'objectif est alors d'empêcher les Haïtiens d'entrer sur le territoire américain où ils pourraient bénéficier des lois fédérales en vigueur. De fait, sur 21600 demandeurs d'asile interceptés entre 1981 et 1991, seuls 28 ont échappé à une reconduite directe en Haiti. Enfin, le Kennebunkport Order de Bush (1992) autorise les garde-côtes à refouler systématiquement les Haïtiens interceptés en haute mer - au mépris de la Convention relative au statut des réfugiés -, les obligeant, en pleine dictature, à passer par l'ambassade de Portau-Prince pour demander l'asile. Près de $3 \%$ des candidats à l'asile reçoivent une issue favorable à leur demande.

Le fait de déplacer la question hors du territoire états-unien comporte, aux yeux de Washington, l'intérêt de contenir une partie des flux maritimes.

22. Source: US Department of Homeland Security.

23. Stepick A., 1982, "Structural Determinants of Haitian Refugee Movement: Different Interpretations", Occasional Papers Series Dialogue, n4, Miami, Latin American and Caribbean Center. 
C'est ce modèle que la Commission européenne a reproduit en 2002 en préconisant l'instauration des procédures d'entrées protégées (PEP) ${ }^{24}$. Ce projet fait écho à la proposition britannique de créer des lieux de rétention des demandeurs d'asile en attente de visas dans la presque totalité des pays européens et aux abords de l'Europe, dans les Etats riverains. Cette idée " complète » la notion de pays sûrs ou de pays tiers sûrs, évoquée précédemment.

En réaction à ce dispositif, les Haitiens diversifient leurs routes et leurs modes de migration, en utilisant diverses îles de la Caraïbe comme espaces de transit plus ou moins durables, qu'il s'agisse de la République dominicaine, de Saint-Martin/Sint Maarten, des Départements français d'Amérique, des Turks et Caicos ou des Bahamas 25. En contenant partiellement les flux, le dispositif maritime états-unien aboutit, de fait, à un transfert indirect de la responsabilité de leur gestion aux pays de transit, qui doivent désormais gérer une présence immigrée durable à laquelle ils ne sont pas préparés. Au cours du temps, les Bahamas - archipel situé aux portes des Etats-Unis - se sont progressivement mués en espace de rétention d'une population d'origine haïtienne qui représente actuellement le quart des 300000 habitants du territoire, mais dont les autorités refusent de reconnaître officiellement la présence. Signe de cette fonction de «sas» assignée aux Bahamas, le centre de détention de Carmichael Road concentre, pour une période plus ou moins longue, des migrants dont les conditions de vie sont particulièrement éprouvantes, avant de les renvoyer dans le pays d'origine. Bien que la pression exercée sur ce territoire par les Etats-Unis soit indéniable, on ne peut néanmoins pas parler d'《externalisation » dans la mesure où il n'y a pas d'accord formel entre les deux gouvernements.

\section{L'expérience migratoire subsaharienne}

Aujourd'hui, face aux difficultés croissantes d'immigration légale, les migrants ouest-africains tendent aussi à diversifier leurs stratégies spatiales. A la différence des trajectoires caribéennes, les routes terrestres ont dans un premier temps primé sur les routes maritimes du fait de la configuration continentale de l'Afrique. Deux routes ont alors été empruntées par les migrants ouest-africains pour rejoindre l'Europe, l'une transitant par la Libye et rejoignant l'Italie, l'autre conduisant en Espagne via le Maroc.

Cependant, dès 2004, un « accord de coopération en matière de flux clandestins » est signé entre l'Italie et la Libye, cette dernière étant considérée

24. En 2001, la Commission a commandé une étude sur «l'examen des demandes d'asile à l'extérieur de l'Union". Cette étude remise en décembre 2002 recommande que les Etats membres envisagent des «modes d'entrées protégées » qui soient « complémentaires» des régimes d'asile existants. Cette étude de faisabilité a été réalisée par le Danish Center for Human Rights et le Danish Refugee Council.

25. Audebert C., op. cit. 
comme une «zone de sas ». En décembre 2007, le processus est renforcé par un nouvel accord italo-libyen de coopération contre l'immigration clandestine. Cet accord prévoit des patrouilles mixtes, formées de militaires libyens et de policiers italiens, qui patrouillent à la fois dans les eaux libyennes et internationales. L'objectif est de rechercher en mer les embarcations des candidats à l'immigration en Europe et surtout, en amont, d'intercepter les bateaux qui embarquent des migrants sur les plages libyennes de Zuwarah, de Zaniyah et de Miswatah notamment. A partir d'un centre opérationnel dont le siège est à Tripoli, la mer est surveillée par radar et par satellite jusqu'aux côtes italiennes.

Au même moment ${ }^{26}$, l'Union européenne se dote d'une agence de surveillance des frontières en Méditerranée, nommée FRONTEX (Agence européenne pour la gestion de la coopération opérationnelle aux frontières extérieures des Etats membres de l'Union européenne). Cette agence a pour mission de coordonner la coopération entre les Etats membres en matière de gestion des frontières extérieures, d'assister les Etats membres pour la formation des garde-frontières nationaux, et de fournir aux Etats membres l'assistance technique et l'appui nécessaire pour organiser des opérations de retour conjointes.

Ces nouveaux moyens de contrôle incitent les migrants ouest-africains, et les Sénégalais en particulier, à privilégier la route par le Maroc, au Nord duquel l'enclave de Ceuta et Mellila devient un tremplin vers l'Europe.

L'analyse des données frontalières des postes terrestres de Kidira ${ }^{27}$, à l'extrémité est du Sénégal, ville frontière avec le Mali sur la route de la Libye, d'une part, et de Rosso ${ }^{28}$, ville frontière avec la Mauritanie, sur la route du Maroc, d'autre part, confirme ces mutations et souligne le rôle de transit du Sénégal entre l'Afrique de l'Ouest et l'Europe.

Jusqu'en 2003, les ressortissants de différents pays ouest-africains qui souhaitent rejoindre l'Europe via le Sénégal privilégient la voie aérienne par l'aéroport international L.S. Senghor de Dakar; mais, en 2004, l'instauration du visa de transit aéroportuaire au Sénégal limite les possibilités d'embarquer pour l'Europe. Dès lors, certains sont tentés d'emprunter la route transsaharienne de l'intérieur, ce qui peut expliquer l'augmentation du nombre de sorties les concernant aux postes de Kidira et de Rosso. Toutefois, en 2005, le nombre de sorties de ressortissants ouest-africains, y compris de Sénégalais, augmente surtout au poste de Rosso. Cela traduit notamment un redéploiement de la voie libyenne vers la voie marocaine, après 2004 .

26. Créée en octobre 2004 et opérationnelle en octobre 2005.

27 . Registre des postes frontaliers de Kidira, entrées et sorties, 2000 à 2005.

28. Registre des postes frontaliers de Rosso, entrées et sorties, 2000 à 2005. 
Le renforcement progressif par les autorités marocaines et espagnoles de la surveillance du détroit de Gibraltar et le drame de Ceuta et Melilla en octobre 2005, incitent les candidats à l'émigration à se tourner vers la voie maritime atlantique. Le livre noir de Ceuta et Melilla explique comment ces événements sont une conséquence de la politique de l'Union européenne visant à sécuriser ses frontières extérieures et à contraindre les candidats à la migration à rester dans leur pays ${ }^{29}$. Il rappelle ainsi les enjeux pour les pays du Sud de l'externalisation du contrôle des frontières extérieures de l'Europe.

En 9 mois, d'octobre 2005 à mai 2006, les routes migratoires se sont déplacées du Sahara à l'Atlantique et les lieux d'embarquement ont glissé de Dahla, au Maroc, à Diogué au Sud du Sénégal, en passant par Layoun ou Nouadhibou, en Mauritanie ${ }^{30}$. Le récit d'un migrant sénégalais témoigne de ce glissement spatial le long des côtes africaines de l'Atlantique Nord :

«Je connaissais avant la possibilité de prendre l'avion. J'ai essayé par deux fois de passer par le Maroc sans succès. C'est alors que la route maritime m'a été proposée [...]. C'est en Mauritanie alors que je faisais la pêche que des jeunes pêcheurs comme moi sont partis en Espagne et leurs échos nous parvenaient à chaque fois comme quoi ils sont rentrés dans ce pays [...]. Je suis alors revenu ici au Sénégal pour me préparer, chercher un peu plus de moyens financiers pour partir de la Mauritanie [...]. J'ai essayé de passer par deux fois par là sans succès et, à chaque fois, c'est la marine marocaine qui nous faisait revenir. Je suis alors revenu au Sénégal dans mon village. De là, des parents et amis partis par D. (Casamance) et arrivés aux Canaries m'ont souvent téléphoné pour me demander de tenter cette voie. Finalement, je suis parti de D. (Casamance); nous avons trouvé d'autres candidats et c'est là que la vraie pirogue qui nous a amenés était amarrée. La pirogue contenait 150 personnes [...]. Plusieurs nationalités composaient le groupe: des Sénégalais, Bissao, Gambiens, Guinéens de Conakry et des Maliens. [...] Le voyage a duré huit jours. A quelque un ou deux kilomètres du rivage (des côtes des îles Canaries) un hélicoptère de la marine espagnole survola notre embarcation tout en nous donnant des consignes de navigation puis un bateau est arrivé pour remorquer la pirogue jusqu'au quai. »

T.B., Département de Sedhiou, Sénégal, août 200731

29. Migreurop, Guerre aux migrants : le livre noir de Centa et Melilla, 2006.

30. Gonin P., Robin N., «Les routes du transit par le Sénégal », Immigration, transit rétention, Colloque de l'IRENAM, Marseille, novembre 2006.

31 . Extraits d'entretiens réalisés au Sénégal en août 2008 auprès de candidats à l'émigration, arrivés aux îles Canaries et qui ont fait l'objet de procédures de réadmission au Sénégal. Projet de recherche financé par l'UNESCO. 
Cette maritimisation des trajectoires traduisant la diversification des stratégies migratoires n'est pas sans rappeler l'expérience des centaines de milliers de boat people caribéens ayant tenté de rejoindre les Etats-Unis au cours des quatre dernières décennies. Au total, 30000 candidats à l'immigration seraient arrivés aux Canaries au cours de l'année 2006 ; selon les autorités espagnoles ${ }^{32}$, la majorité d'entre eux était de nationalité sénégalaise.

Les départs ont commencé au cours du premier trimestre 2006 et se sont intensifiés en juillet et en août, les mois les plus favorables à la navigation vers les îles Canaries, comme le rappellent les capitaines de pêche : "C'est aux mois de juillet et août que l'on a les meilleures conditions; la mer est calme, la température est bonne, il n'y a que la pluie. Les mois les plus dangereux sont de mars à mai 33 ».

En réponse à cette nouvelle émigration par voie maritime, en juin 2006, à la demande de l'Espagne, Frontex étend son «dispositif de contrôle de l'émigration clandestine » aux eaux de l'Atlantique nord, au large de la Mauritanie d'abord, puis du Sénégal ensuite. L'objectif est d'intercepter les embarcations suspectées de faire route vers l'archipel espagnol des Canaries.

Malgré cette surveillance aérienne et maritime accrue, les départs se poursuivent en $2007^{34}$ et continuent en 2008, comme en témoignent régulièrement les interceptions d'embarcations, médiatisées par les autorités dans l'intention de dissuader les candidats potentiels.

\section{La sophistication des dispositifs, entre transfert de responsabilité et délocalisation spatiale}

Les dynamiques spatiales décrites et les dispositifs mis en place tant du côté africain que du côté caribéen constituent le prélude à une autre étape, celle d'une externalisation offshore. Celle-ci est doublée d'une délocalisation des moyens et infrastructures dans les dépendances territoriales ultra-marines ayant une position géographique stratégique en Méditerranée, dans l'Atlantique nord et dans la Caraïbe. Face à la maritimisation de l'émigration depuis les côtes du Sénégal et d'Haïti, l'Europe et les Etats-Unis associent donc deux stratégies. L'une vise à la distanciation juridique (action hors du

32. Cette estimation a été établie en fonction de la langue parlée par les candidats à l'immigration lors d'entretiens réalisés en Espagne par des policiers sénégalais.

33. Extraits d'entretiens réalisés au Sénégal en août 2008 auprès de candidats à l'émigration, arrivés aux îles Canaries et qui ont fait l'objet de procédures de réadmission au Sénégal. Projet de recherche financé par l'UNESCO.

34 . 5600 personnes sont arrivées aux îles Canaries entre les mois de janvier et de juillet 2007. Selon les autorités espagnoles, la plupart des pirogues provenaient du Sénégal. La même source précise que les pirogues parties de Mauritanie, de Guinée Bissau ou de Gambie avaient également été construites au Sénégal. 
cadre législatif des pays du Nord) et/ou géographique (éloignement des pays du Nord) du traitement de la migration. L'autre a pour objectif le partage des responsabilités de la gestion des flux avec les pays de transit au Sud.

\section{L'externalisation offshore : un transfert de responsabilité}

L'expression d' « externalisation offshore » du contrôle des flux migratoires traduit l'importation des méthodes, des outils et d'une partie des ressources humaines destinés à la mise en œuvre de l'accord FRONTEX, signé entre l'Espagne et le Sénégal en août 2006 35. Effectivement, ce dispositif prévoit le renforcement des mesures de surveillance de la côte par des patrouilles conjointes, l'objectif étant d' «intercepter les pirogues d'émigrés clandestins 36 ». En outre, pour assurer sa mission, FRONTEX bénéficie de moyens logistiques : deux vedettes, un avion et un hélicoptère espagnols et un avion italien. Ainsi, l'identification des candidats à l'émigration vers le territoire européen et la responsabilité des résultats sont déléguées aux autorités sénégalaises, à la différence $\mathrm{du}$ cas de figure caribéen précédemment évoqué où les Etats-Unis conservent le contrôle total du dispositif logistique.

Ce nouveau transfert de responsabilité est complémentaire de la stratégie européenne de l'externalisation de l'asile, imaginée en 2004 sous la forme des PPR (Programmes de protection régionaux) et de la réinstallation des réfugiés. La clé de voûte de ce système résiderait dans la mise en place d'espaces de rétention des demandeurs d'asile dans les pays de départ ou dans des pays tiers - pays de transit comme la Lybie, la Mauritanie, l'Algérie 37, voire l'Ukraine. Le nouveau schéma vise à la fois à réduire les coûts du traitement de l'asile en Europe en développant les capacités d'accueil dans les pays extracommunautaires et à contenir les flux en assignant à des Etats extérieurs à l'Union européenne une fonction de filtrage des demandes d'asile. Il est complété par la négociation d'accords de réadmission de migrants majoritairement subsahariens avec le Maroc, le Sénégal, la Gambie et la Guinée, notamment, au mépris du principe de non-refoulement. Ainsi, au cours des années 2006 et 2007, plusieurs milliers de migrants ayant atteint les côtes des îles Canaries

35. Cet accord s'inscrit dans le prolongement de l'accord signé quelques mois auparavant entre l'Espagne et la Mauritanie.

36. En juin 2007, les autorités espagnoles et sénégalaises ont signé un nouvel accord qui prolonge FRONTEX d'un an; il a été de nouveau prolongé d'un an par un accord signé par le ministre de l'Intérieur sénégalais et son homologue espagnol, en mai 2008.

37. "Actualisant la législation régissant l'entrée et la circulation des étrangers en Algérie, un projet de loi sera soumis aux débats lors de la session parlementaire en cours. Un projet de loi élaboré par le ministère de l'intérieur et des collectivités locales conformément aux directives du conseil interministériel du 14 juin 2005 demandant "la criminalisation de l'immigration illégale". Il est préconisé de créer par voie réglementaire des centres d'accueil destinés à l'bébergement provisoire des ressortissants étrangers en situation irrégulière. L'article 36 de ce projet indique que ce placement peut être ordonné par arrêté $d u$ wali territorialement compétent pour une période de 30 jours renouvelable », Le Soir d'Algérie, 25 mars 2008. 
ont été rapatriés au Sénégal, après un temps de détention dans des camps. Ces réadmissions forcées sont organisées alors que le texte qui les prévoit fait encore l'objet de négociations entre l'Espagne et le Sénégal.

Dans ses évolutions les plus récentes, la stratégie européenne reproduit à une ou deux décennies d'intervalles une stratégie bien éprouvée outreAtlantique. Dans la Caraibe, des accords de réadmission bilatéraux (bilateral interdiction agreements) ont été signés par la superpuissance avec l'ensemble des Etats de la région, y compris le régime castriste de Cuba en 1995 38. La même année, le nombre de Cubains admis temporairement aux Etats-Unis a alors été divisé par deux. Le processus d'externalisation offshore impulsé par Washington s'est parallèlement décliné sous la forme d'une externalisation de l'asile par le transfert des demandeurs d'asile haïtiens vers des pays tiers de la région afin d'éviter de les accueillir directement sur le sol états-unien.

Les relations diplomatiques, militaires, commerciales et financières asymétriques entre certains territoires du bassin caribéen et le puissant voisin $\mathrm{du}$ Nord ${ }^{39}$ ont été un atout déterminant pour convaincre les premiers d'accueillir temporairement les réfugiés. Ainsi, au cours des deux mois ayant suivi le coup d'Etat militaire haïtien de 1991, 4500 réfugiés maritimes ont été interceptés, dont 80 \% ont été détenus sur les navires des gardes-côtes. Mais l'explosion ultérieure des flux a obligé Washington à négocier leur réinstallation dans des pays tiers volontaires, le temps de traiter les demandes. Outre Belize, le Vénézuela et Trinidad-et-Tobago, le Honduras a accepté d'accueillir 40000 Haïtiens en échange d'une aide financière états-unienne substantielle. Un temps suspendu, le traitement offshore des demandes d'asile a été rétabli par Clinton en 1994 sous la pression des lobbies états-uniens : les îles Turks et Caicos et les eaux territoriales jamaïcaines ont été à leur tour mises à contribution.

\section{La délocalisation spatiale}

Cependant, la notion de «méditerranée américaine » prend véritablement une autre dimension avec une stratégie alternative des Etats-Unis mise en œuvre à partir de 1991 consistant, parallèlement à l'appel à des pays tiers, à utiliser ses propres bases militaires (en plus de ses territoires ultra-marins) pour « accueillir » les boat people. Un camp de rétention a été alors ouvert sur la base militaire états-unienne de Guantanamo, dans l'Est de Cuba... et surtout en face d'Haïti. La base a concentré jusqu'à 34000 demandeurs d'asile. Les conditions de vie y ont été difficiles, en particulier pour les migrants séro-

38. Singulièrement, seul le gouvernement haïtien d'Aristide mettra fin à son accord bilatéral de réadmission avec les Etats-Unis en 1994 pour mettre Washington face à l'obligation de nonrapatriement stipulée par sa Convention relative au statut des réfugiés.

39. Maingot A., The United States and the Caribbean: Challenges of an Asymetrical Relationship, Boulder, Westview Press, 1994. 
positifs transférés dans la zone d'enfermement spécifique de Bulkeley et dont la demande d'asile aux Etats-Unis a été systématiquement rejetée.

Guantanamo est de fait longtemps resté un no man's land, où la législation états-unienne ne s'appliquait que de manière aléatoire. En dépit de l'examen souvent peu rigoureux des demandes, et du fait que $7 \%$ des dossiers aient été bâclés ou égarés, l'arrivée de fonctionnaires indépendants a permis à un tiers des demandes de recevoir une issue favorable. L'expérience de Guantanamo montre que la délocalisation géographique du dispositif de contrôle de l'immigration s'accompagne d'une indéniable militarisation du dispositif. Lors de la réouverture de la base aux réfugiés en 1994, la moitié des 2200 civils présents a été transférée aux Etats-Unis « pour des raisons de sécurité ", tandis que 3200 militaires étaient désormais présents en permanence sur la base, dont 800 spécifiquement affectés au maintien de l'ordre.

Les possessions territoriales états-uniennes dans la Caraïbe ont également pu ponctuellement être mobilisées dans l'optique de contenir les flux de migrants maritimes caribéens hors du territoire métropolitain des Etats-Unis. Par exemple, la base militaire de Fort Allen à Puerto Rico a été transformée en centre d'accueil pour les réfugiés cubains et haïtiens entre 1980 et 1983.

Force est donc de constater que la délocalisation spatiale du dispositif de contrôle des flux par l'Union européenne, vingt ans après l'expérience américaine, n'est pas une innovation. L'implantation des camps de rétention et de tri des candidats à l'émigration sur le territoire insulaire européen le plus proche du continent africain apparait comme la reproduction de méthodes déjà expérimentées ailleurs. Les dernières années ont vu la multiplication, dans ces espaces stratégiques, des camps d'accueil des étrangers dans l'optique du traitement des demandes d'admission dans l'Union européenne ou en instance d'expulsion.

Si l'Espagne a mis en place trois centres à proximité du détroit de Gibraltar et un centre à Malaga, elle tend également à développer son dispositif bien au-delà du territoire continental. Outre les deux centres ouverts de Ceuta et Melilla, quatre centres ont vu le jour dans l'archipel des Canaries. La Sicile compte actuellement cinq camps et le petit Etat maltais, dont la position est hautement stratégique, concentre à lui seul sept camps - dont trois ouverts. Cette nouvelle géographie délocalisée des camps est complétée par un dispositif de détection ultra sophistiquée de l'immigration clandestine : trois radars sont désormais opérationnels en Andalousie, deux aux Canaries et un sur l'île italienne de Pantelleria ${ }^{40}$. 
Ainsi, en transformant certains pays du Sud en « opérateurs de contrôle » de l'émigration vers les pays du Nord et en érigeant les rivages européens au contact de l'Afrique en "postes avancés » de l'Europe forteresse, les pays européens sont entrés dans une logique de criminalisation des migrants, au mépris du droit des personnes à circuler et à quitter le pays où elles se trouvent, et de militarisation du contrôle des flux migratoires, au mépris du droit international.

A l'aune des expériences de mise en place des dispositifs frontaliers américains et européens visant au contrôle de l'émigration caribéenne et subsaharienne, la transposition à la géopolitique de la notion d'« externalisation » dans son acception économique est-elle en définitive pertinente ? L'instauration des visas d'entrée dès les pays de départ, la nouvelle fonction de "sas migratoire » assignée aux territoires de transit, et la sophistication des dispositifs face au redéploiement des routes migratoires révèlent toutes la volonté de mise à distance des dispositifs de contrôle migratoire vis-à-vis des espaces européen et nord-américain.

FRONTEX apparaît en Europe comme l'exemple le plus abouti de ce processus d'externalisation, en symbolisant le passage de l'externalisation simple à l'externalisation offshore. La délégation de fonction associe la délégation de compétence à l'importation des méthodes, des outils et du travail. Elle est assortie d'une gestion déléguée (identification des passagers en direction des pays du Nord, responsabilité des résultats), l'objectif étant d'éloigner des territoires et des citoyens européens et états-uniens les moyens mis en œuvre pour obtenir des résultats. La logique quantitative qui sous-tend ces stratégies se met en même temps à l'abri - au moins partiellement - de la législation en vigueur des pays du Nord avec laquelle elle rentre en contradiction.

Ainsi, tant dans ses logiques explicatives (baisse des coûts opérationnels, flexibilité, amélioration de la qualité du service, recherche d'un cadre législatif avantageux) que dans sa dimension évolutive (extension du processus, nouvelles exigences), l'acception économique de l'externalisation comporte un certain nombre d'analogies et de correspondances avec celle appliquée dans le domaine de la réévaluation des dispositifs frontaliers des « forteresses » européenne et nord-américaine. Néanmoins, son application trouve ses limites dans l'utilisation de plus en plus répandue de la position géostratégique des territoires ultramarins des Etats-Unis ou de l'Union européenne pour contrôler les flux. Il convient alors de parler d'« extériorisation » des dispositifs, plutôt que d'« externalisation».

La mise en perspective des expériences subsaharienne et africaine à quelques décennies d'intervalles nous apporte deux enseignements. Premièrement, 
la politique de l'Union européenne n'est pas véritablement innovante et ne fait que reproduire avec quelques variantes des dispositifs à l'œuvre outreAtlantique depuis plusieurs décennies. Or, deuxièmement, ces dispositifs ont montré leur relative inefficacité depuis trente ans dans les Amériques - comme en témoigne la présence sans cesse croissante d'une communauté haïtienne désormais forte d'un demi-million de personnes en Floride - et il est peu probable qu'il en soit différemment pour l'Union européenne à terme. La politique du chiffre semble vouée à l'échec.

Il serait probablement plus efficace - mais aussi plus exigent en termes de réflexion systémique sur le long terme - de s'orienter vers une politique plus globale prenant notamment en compte les causes profondes et structurelles des mouvements migratoires ainsi que la complexité croissante de leurs dynamiques sociales et spatiales. La complexification des parcours migratoires, la réversibilité des migrations, la dimension collective des stratégies et, plus généralement, la mise en place de véritables champs migratoires internationaux rendent caduques les approches binaires opposant pays d'émigration et pays d'immigration qui inspirent encore dans une large mesure les politiques migratoires des pays du Nord. 